Michael Ridley

Western University, London, Ontario, Canada

University of Guelph, Guelph, Ontario, Canada

\title{
AUTONOMOUS INFORMATION BEHAVIOUR: TOWARDS A CONCEPTUAL MODEL
}

\begin{abstract}
Autonomous information behaviour (AIB) proposes that autonomous intelligent agents exhibit information behaviour characteristics (i.e. "need, seek, manage, give, and use information in different contexts" Fisher, Erdelez, \& McKechnie, 2005, p. xix) similar to that in humans. A preliminary conceptual model of AIB, derived from Wilson (2016) and Domingos (2015), is presented. Understanding autonomous information behaviour is important given the increasing prevalence of algorithmic decision-making in everyday life.
\end{abstract}

\section{Introduction}

Models of information behaviour (IB) are typically human-centric (Bates, 2010; Case \& Given, 2016; Fisher et al., 2005; Wilson, 2010). Autonomous information behaviour (AIB) proposes that artificial intelligence, or more specifically autonomous intelligent agents (AIA) using machine learning, share similar characteristics as human information behaviour (HIB). As with humans, AIAs "need, seek, manage, give, and use information in different contexts" (Fisher et al., 2005, p. xix).

Understanding AIB is important because of the prevalence of algorithmic decisionmaking in many aspects of everyday life and because "the danger is not so much in delegating cognitive tasks, but in distancing ourselves from - or in not knowing about - the nature and precise mechanisms of that delegation" (de Mul \& van den Berg, 2011, p. 59). The IB field, because of its focus on human information seeking and use, has exhibited only a limited interest in autonomous agents and their information behaviour (Ingwersen \& Järvelin, 2005; G. Liu, 2011; Steels \& Brooks, 1995). Locating AIB in the existing IB field will help to address that isolation and permit AIB and HIB to inform each other.

\section{Autonomous Intelligent Agents}

Fully autonomous intelligent agents are exemplars of AGI (artificial general intelligence) (Boden, 2016; Bostrom, 2014; Primiero, 2017) and none currently exist. However, there are AIAs in specialized, narrowly defined domains (e.g. games, drug discovery). It is an emergent area just as is the whole field of AI. As a result, successes and failures are abundant and it is easy to be selective from either to support an argument about the state of AI. AIAs are developing, imperfect, narrowly implemented, tenuous, and yet rapidly evolving. Their autonomy is limited and often entwined with or even dependent upon the agent designers. Given the emergent nature of AIA, a reasonable challenge to the concept of AIB is that we are examining the IB of the 
agent designers rather than that of the agent. However, the course of AIA research and development is toward increased autonomy in wider domains suggesting that examining nascent AIB will be valuable as an early indicator of future directions.

A survey of AIA characteristics resulted in an extensive list: autonomy, social ability, reactivity, pro-activeness; notions of knowledge, belief, intention, and obligation; mobility, veracity, benevolence, and rationality (Wooldridge \& Jennings, 1995). Maes simplified this focusing on only two features: competence and trust (Maes, 1994). Gaming has long been a focus for AI research (Yannakakis \& Togelius, 2018). While games such as Go and Dota 2 are limited and constrained domains, they provide a context to observe how autonomous intelligent agents gather, assess, synthesize, and utilize information thereby engaging in various information behaviours.

Go provides the challenge of complexity within a simple set of rules. Of particular interest is AlphaGo Zero which learned to play with only the rules of the game and the ability to play against itself (i.e. no access to historical games or human expertise or guidance) (Silver et al., 2016, 2017). In defeating Go world champion Lee Sedol, the developers asserted AlphaGo Zero that exhibited "genuine moments of creativity" and was "no longer constrained by the limits of human knowledge" (Knapton, 2017).

Dota 2, a multiplayer battle strategy game, provides a different set of challenges, arguably more complex and nuanced than Go because of the long time horizons, partiallyobservable status (e.g. fog can temporarily obscure the board), continuous action space, and continuous observation space ("OpenAI Five," 2018). After a 2018 loss where OpenAI Five exhibited good tactics but strategic weaknesses (Simonite, 2018), the agent recently defeated the Dota 2 world champions "deftly navigating strategic decisions and racing to press its advantages with uncannily good judgment” (Piper, 2019).

\section{HIB and AIB}

One approach to understanding AIB is to use HIB as a benchmark. The IB focus on specific contexts, roles or situations has resulted in a lack of generalization or extrapolation to a more general theory (Case \& Given, 2016; Wilson, 1994, 2010). IB has been "small-scale and fragmented, providing a snapshot view of phenomena" (Ford, 2015, p. 239). While a number integrated IB models have been proposed (Godbold, 2006; J. Liu, 2017; Nahl, 2007; Narayan, 2010; Robson \& Robinson, 2013; Shenton \& Hay-Gibson, 2012; Zhang \& Soergel, 2014), it is Wilson's model that most approximates a general theory of IB and can serve as a benchmark for AIB (Wilson, 1981, 1999, 2016).

Wilson's model has seven foundational concepts: person-in-context, information need, activating mechanisms, intervening variables, information seeking behaviours, information processing, and information use. These broad concepts and their interactions are sufficiently inclusive to account for the IB theories that focus on specific contexts and roles (Wilson, 2016). The interactions among these concepts are non-linear. Activating mechanisms, intervening variables, and information seeking behaviours interact throughout an IB process or event. Information need, while an initiating event, is also a context that is refined throughout the information seeking and use process (Cole, 2012). These concepts and interactions can be used as a general model of AIB allowing for the identification of contexts, actions, and outcomes specific to AIAs. 
In addition to applying Wilson's HIB concepts, three core elements common to all algorithmic models are identified: representation, evaluation, and optimization (Domingos, 2015). Representation is how the model is expressed (i.e. symbols, logic, statistical) as well as how the data is structured and understood. Evaluation is the scoring function of the model and how well the model fits the data. Optimization is the process that searches for the best set of parameters using specific testing and refinement techniques. While these elements are implemented differently according to the concept of algorithmic decision-making that frames the AIA, all are present in AIB.

Figure 1 illustrates the preliminary Autonomous Information Behaviour model, integrating the Wilson HIB model with the key algorithmic components from Domingos.

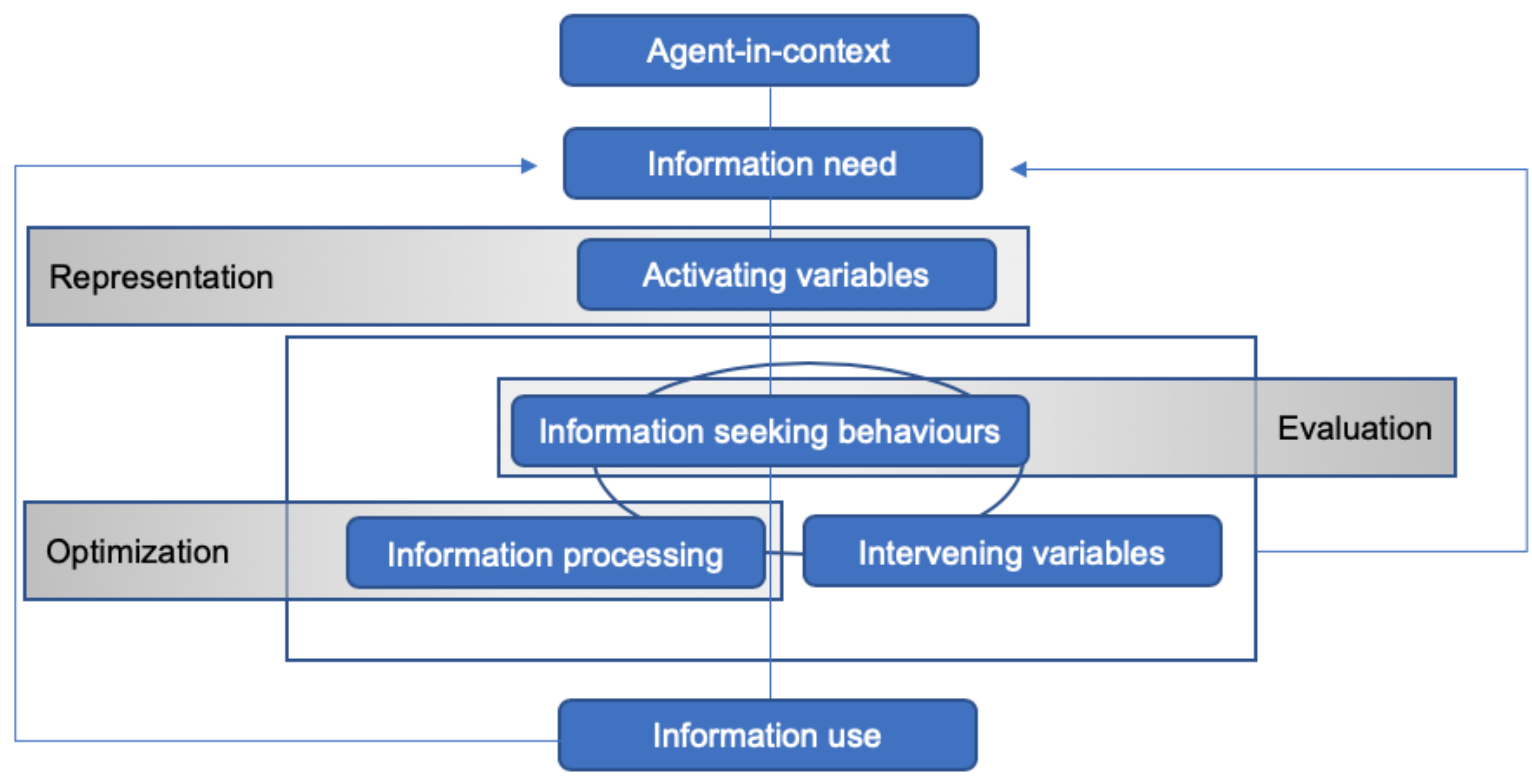

Figure 1: Autonomous Information Behaviour Model

This integrated or layered model highlights the similarities and differences between HIB and AIB. The core of any human information behaviour model is information seeking and this focus is shared with AIB: "It has been largely claimed that most, if not all, of artificial intelligence is really just search. Almost every AI problem can be cast as a search problem, which can be solved by finding the best (according to some measure) plan, path, model, function, etc." (Yannakakis \& Togelius, 2018, p. 39). However, search is implemented differently in AIB.

For example, in HIB information processing and information representation occur together in a later stage in the model (e.g. the loop that ties together information seeking, information processes, and intervening variables). In AIB, information representation occurs at an earlier stage and is dictated by the specific model to be utilized. The iterative process of HIB regarding processing and representation contrasts with the sequential approach of AIB. AlphaGo Zero uses an ensemble approach incorporating, at various stages, neural networks, reinforcement learning, and decision trees to mitigate the limitations of sequential processing (Silver et al., 2016, 2017).

The intervening variables of the Wilson model correspond to the specific algorithms employed by the machine learning model. For example, OpenAI Five uses proximal policy 
optimization (“OpenAI Five," 2018; Schulman, Wolski, Dhariwal, Radford, \& Klimov, 2017), a more flexible and responsive search algorithm as part of the reinforcement learning model (Sutton \& Barto, 2018) common in AI gaming agents (Mnih et al., 2015). The algorithm allows for the refinement of parameters (optimization) that is more effective for dynamic environments (e.g. online battle strategy gaming). Selecting the appropriate algorithm or set of algorithms is influenced by such factors as the nature of the data being processing and the available computational capacity.

However, all these algorithms are responsive to a defined cost function or utility function which are "the training signal of any machine learning algorithm as it offers a measure of goodness of the representation we have" (Yannakakis \& Togelius, 2018, p. 32). This cost or utility function, defined variously as rewards, correlations or clusters, is the critical component in the evaluation stage. It is the basis of the feedback or backpropagation loop that enables optimization. In AIB, as in HIB, the aspects of information behaviour that address assessment, veracity, and strategy are central in understanding information seeking and information use.

\section{Conclusion}

The proposed conceptual model of autonomous information behaviour is a starting point for a more rigorous exploration. However, by using Wilson's general model of human information behaviour as a template upon which to apply Domingos elements, a preliminary model of AIB can be outlined in which autonomous intelligent agents, in different contexts, respond to information needs, exhibit similar or analogous strategies, techniques, and actions in information seeking and information processing, and utilize information in various ways. While autonomous agents are currently restricted to limited domains and contexts, understanding their nascent AIB is important if we are to accept and trust their increasing role in everyday decision-making.

\section{Reference List:}

Bates, M. J. (2010). Information behavior. In M. J. Bates \& M. N. Maack (Eds.), Encyclopedia of library and information sciences (3rd ed., pp. 2381-2391). Boca Raton, FL: CRC Press.

Boden, M. A. (2016). AI: Its nature and future. Oxford: Oxford University Press.

Bostrom, N. (2014). Superintelligence: Paths, dangers, strategies. Oxford: Oxford University Press.

Case, D. O., \& Given, L. M. (2016). Looking for information: A survey of research on information seeking, needs and behavior (4th ed.). Bingley, UK: Emerald Group Publishing.

Cole, C. (2012). Information need: A theory connecting information search to knowledge formation. Medford, New Jersey: American Society for Information Science and Technology.

de Mul, J., \& van den Berg, B. (2011). Remote control: Human autonomy in the age of computer-mediated agency. In M. Hildebrandt \& A. Rouvroy (Eds.), Law, human agency, and autonomic computing (pp. 46-63). Abingdon: Routledge.

Domingos, P. (2015). The master algorithm: How the quest for the ultimate learning machine will remake our world. New York: Basic Books. 
Fisher, K. E., Erdelez, S., \& McKechnie, L. (Eds.). (2005). Theories of information behavior. Medford, NJ: American Society for Information Science and Technology.

Ford, N. (2015). Introduction to information behaviour. London: Facet Publishing.

Godbold, N. (2006). Beyond information seeking: Towards a general model of information behaviour. Information Research, 11(4). Retrieved from http://www.informationr.net/ir/11-4/paper269.html

Ingwersen, P., \& Järvelin, K. (2005). The turn: Integration of information seeking and retrieval in context. Dordrecht: Springer.

Knapton, S. (2017, October 18). AlphaGo Zero: Google DeepMind supercomputer learns 3,000 years of human knowledge in 40 days. The Telegraph. Retrieved from http://www.telegraph.co.uk/science/2017/10/18/alphago-zero-google-deepmindsupercomputer-learns-3000-years/amp/

Liu, G. (2011). The application of intelligent agents in libraries: A survey. Program: Electronic Library and Information Systems, 45(1), 78-97. https://doi.org/10.1108/00330331111107411

Liu, J. (2017). Toward a unified model of human information behavior: An equilibrium perspective. Journal of Documentation, 73(4), 666-688. https://doi.org/10.1108/JD-06$\underline{2016-0080}$

Maes, P. (1994). Agents that reduce work and information overload. Communications of the ACM, 37(7), 30-40. https://doi.org/10.1145/176789.176792

Mnih, V., Kavukcuoglu, K., Silver, D., Rusu, A. A., Veness, J., Bellemare, M. G., ... Hassabis, D. (2015). Human-level control through deep reinforcement learning. Nature, 518(7540), 529-533. https://doi.org/10.1038/nature14236

Nahl, D. (2007). Social-biological information technology: An integrated conceptual framework. Journal of the American Society for Information Science and Technology, 58(13), 20212046. https://doi.org/10.1002/asi.20690

Narayan, B. (2010). Towards developing an integrated model of information behaviour (PhD Dissertation, Queensland University of Technology). Retrieved from https://eprints.qut.edu.au/33252/1/Bhuvaneshwari_Lakshminarayanan_Thesis.pdf

OpenAI Five. (2018, June 25). Retrieved March 24, 2019, from OpenAI website: https://openai.com/blog/openai-five/

Piper, K. (2019, April 13). AI triumphs against the world's top pro team in strategy game Dota 2. Retrieved April 17, 2019, from Vox website: https://www.vox.com/2019/4/13/18309418/open-ai-dota-triumph-og

Primiero, G. (2017). Algorithmic iteration for computational intelligence. Minds and Machines, 27(3), 521-543. https://doi.org/10.1007/s11023-017-9423-8

Robson, A., \& Robinson, L. (2013). Building on models of information behaviour: Linking information seeking and communication. Journal of Documentation, 69(2), 169-193. https://doi.org/10.1108/00220411311300039

Schulman, J., Wolski, F., Dhariwal, P., Radford, A., \& Klimov, O. (2017). Proximal policy optimization algorithms. ArXiv:1707.06347 [Cs]. Retrieved from http://arxiv.org/abs/1707.06347

Shenton, A. K., \& Hay-Gibson, N. V. (2012). Information behaviour meta-models. Library Review, 61(2), 92-109. https://doi.org/10.1108/00242531211220735 
Silver, D., Huang, A., Maddison, C. J., Guez, A., Sifre, L., van den Driessche, G., ... Hassabis, D. (2016). Mastering the game of go with deep neural networks and tree search. Nature, 529(7587), 484-489. https://doi.org/doi.org /10.1038/nature16961

Silver, D., Schrittwieser, J., Simonyan, K., Antonoglou, I., Huang, A., \& Guez, A. (2017). Mastering the game of Go without human knowledge. Nature, 550, 354-359. https://doi.org/10.1038/nature24270

Simonite, T. (2018, August 23). Pro gamers fend off Elon Musk-backed AI bots-for now. Wired. Retrieved from https://www.wired.com/story/pro-gamers-fend-off-elon-musks-aibots/

Steels, L., \& Brooks, R. A. (1995). The artificial life route to artificial intelligence: Building embodied, situated agents. Hillsdale, NJ: Lawrence Erlbaum Associates.

Sutton, R. S., \& Barto, A. G. (2018). Reinforcement learning: An introduction (2nd ed.). Cambridge: MIT Press.

Wilson, T. D. (1981). On user studies and information needs. Journal of Documentation, 37(1), 3-15. https://doi.org/10.1108/eb026702

Wilson, T. D. (1994). Information needs and uses: Fifty years of progress? In B. C. Vickery (Ed.), Fifty years of information progress: a Journal of Documentation review (pp. 1551). London: Aslib, The Association for Information Management.

Wilson, T. D. (1999). Models in information behavior research. Journal of Documentation, 55(3), 249-70. https://doi.org/10.1108/EUM0000000007145

Wilson, T. D. (2010). Information behavior models. In M. J. Bates \& M. N. Maack (Eds.), Encyclopedia of library and information sciences (3rd ed., pp. 2391-2400). Boca Raton, FL: CRC Press.

Wilson, T. D. (2016). A general theory of human information behaviour. Information Research, 21(4). Retrieved from http://www.informationr.net/ir/21-4/isic/isic1601.html

Wooldridge, M., \& Jennings, N. R. (1995). Intelligent agents: Theory and practice. The Knowledge Engineering Review, 10(2), 115-152.

Yannakakis, G. N., \& Togelius, J. (2018). Artificial intelligence and games. Cham, Switzerland: Springer.

Zhang, P., \& Soergel, D. (2014). Towards a comprehensive model of cognitive process and mechanisms of individual sensemaking. Journal of The American Society For Information Science, 65(9), 1733-1756. https://doi.org/10.1002/asi.23125 